\title{
Checklist de acessibilidade em ambientes informacionais na Web
}

\author{
Checklist of accessibility in Web informational environments
}

Christiane Gomes dos Santo ${ }^{1}$, Sueny Gomes Léda Araújo ${ }^{1}$, Marckson Roberto Ferreira de Sousa ${ }^{1}$,

Wagner Junqueira de Araújo ${ }^{2}$

${ }^{1}$ Universidade Federal da Paraíba

${ }^{2}$ Universidade de São Paulo

RESUMO: Esta pesquisa aborda os processos de busca, navegação e recuperação da informação pela pessoa com cegueira em ambiente web, focalizando no conhecimento das áreas de recuperação e arquitetura da informação, para o entendimento das estratégias utilizadas por essas pessoas para o acesso à informação na web. Tem como objetivo propor a construção de um instrumento de verificação de acessibilidade, checklist, a ser utilizado para análise do comportamento da pessoa com cegueira em ações de busca, navegação e recuperação em sites e páginas. Trata-se de uma pesquisa de cunho exploratório e descritivo de natureza qualitativa, tendo como metodologia de investigação, o estudo de caso - pela pesquisa estabelecer um estudo específico de simulação de busca, navegação e recuperação da informação utilizando o sistema de síntese de voz, NonVisual Desktop Access, em laboratório de tecnologias assistivas, para fundamentação da construção do checklist para verificação de acessibilidade. Considera-se a confiabilidade da pesquisa efetuada e sua importância para a avaliação da acessibilidade em ambiente web para a melhoria do acesso à informação por pessoas com limitação à leitura, de modo a ser utilizado em análises de verificação de acessibilidade de sites e páginas.

PALAVRAS-CHAVE: Busca de informação.

Acessibilidade na web. Deficiente visual.

Checklist.
ABSTRACT: This research deals with the process of search, navigation and retrieval of information by the person with blindness in web environment, focusing on knowledge of the areas of information recovery and architecture, to understanding the strategies used by these people to access the information on the web. It aims to propose the construction of an accessibility verification instrument, checklist, to be used to analyze the behavior of people with blindness in search actions, navigation and recovery sites and pages. It a research exploratory and descriptive of qualitative nature, with the research methodology, case study - the research to establish a specific study with the simulation of search, navigation and information retrieval using speech synthesis system, NonVisual Desktop Access, in assistive technologies laboratory, to substantiate the construction of the checklist for accessibility verification. It is considered the reliability of performed research and its importance for the evaluation of accessibility in web environment to improve the access of information for people with limited reading in order to be used on websites and pages accessibility check analysis.

KEYWORDS: Information retrieval. Access to information. blindness. Checklist.

\section{Correspondência}

${ }^{1}$ Christiane Gomes dos Santo

Universidade Federal da Paraíba.

João Pessoa, PB.

Email: christiane.gomes.cgs@gmail.com

ORCID: http://orcid.org/0000-0001-7271-0990

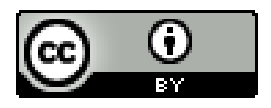

JITA: KE. Architecture 


\section{INTRODUÇÃO}

Para as pessoas com deficiência visual, o avanço tecnológico no campo da informação e comunicação, referente ao aparecimento da web, tem possibilitado significativas mudanças em seu comportamento de busca informacional, devido ao desenvolvimento da tecnologia assistiva para o acesso à informação, com base nos recursos e mecanismos advindos com a tecnologia digital. Considera-se que o texto e som digital são os elementos-chave para o acesso à informação na atualidade, com a utilização dos computadores, por pessoas com deficiência visual. Condição que somente é possível com os softwares de ampliação/contraste e reconhecimento de caracteres para voz, que permitem o acesso a textos, imagens/gráficos descritos e outros softwares.

Com a tecnologia dos softwares de síntese de voz, observou-se um novo marco tecnológico. A sua utilização está permitindo novas possibilidades para o acesso à informação desde a obtenção da leitura e escrita, à produção de produtos digitais acessíveis que estão proporcionando o desenvolvimento/adaptação de textos e livros digitais acessíveis a partir do processo de digitalização convencional ou simples; produção de livros digitais acessíveis utilizando o padrão Digital Accessible Information System (DAISY - tradução livre: Sistema de Informação Acessível Digital); ou mesmo o acesso aos livros eletrônicos (ebooks), e a rede mundial de computadores com a utilização da ferramenta web.

Evidencia-se que a utilização da web por pessoas com deficiência visual compreende especificidades que determinam o processo de busca, navegação e recuperação da informação nesse ambiente. Por exemplo, para que um sistema de síntese de voz possa estabelecer o reconhecimento de caracteres textuais que compõem os conteúdos, organização e estrutura dos sites, é necessário o estabelecimento de uma construção acessível, condição que somente é possível com o seguimento de diretrizes de acessibilidade para a produção de sites em ambiente web. Diante disso, questiona-se: quais os mecanismos que auxiliam no processo de busca, navegação e recuperação da informação por usuários com deficiência visual? Esses mecanismos são efetivamente utilizados? E quais suas condições de uso?

Com esses questionamentos, esta pesquisa objetivou analisar os sistemas de organização de sites com simulações de busca, navegação e recuperação da informação na web, utilizando software de síntese de voz para verificação da acessibilidade. Com os dados obtidos pelas simulações foi possível efetivar a construção de um checklist simples (lista de verificação), que tem por finalidade a sua utilização em processos de avaliação de busca de informação por usuários com cegueira, onde podem ser evidenciadas as estruturas organizacionais de sites, a navegabilidade e a recuperação da informação, implicando na identificação das particularidades das interações do usuário com cegueira - por meio do software de síntese de voz e de sistemas de organização informacional na web oportunizando a verificação das principais dificuldades encontradas por esses usuários no processo de busca informacional em ambiente web. 
A técnica de verificação de inspeções por checklist permite a identificação de problemas gerais e de repetição nas interfaces web, compreendendo um mecanismo que propicia lembrar e/ou solicitar atenções às questões e temas acerca do que se propõe verificar (SALES, 2002). O checklist pode ser construído, de forma geral (descrição de etapas de processos para que não sejam esquecidas), ou de forma específica (lista de itens detalhados que precisam ser abordados). É importante considerar que o checklist não se trata de um conjunto de etapas que precisam ser escolhidas, mas que precisam ser levadas à discussão e à análise de resultados (DOWNEY; BANERJEE, 2010).

Para este estudo, a construção de um checklist corresponde ao fornecimento de procedimentos de ajuda rápida, que permita a verificação da busca da informação na web, a partir da interação do usuário com cegueira e os sistemas de organização de conteúdo informacional, tanto com base nas recomendações de acessibilidade quanto com o uso do software de síntese de voz, possibilitando a produção de resultados uniformes e gerais, para a identificação dos problemas de acessibilidade.

Para a concretização desta pesquisa, estabeleceu-se uma metodologia de níveis exploratório e descritivo, centrando-se no conhecimento dos processos de busca, navegação e recuperação da informação, de natureza qualitativa, baseando-se em uma metodologia investigativa determinada pelo estudo de caso único direcionado à construção do checklist para verificação da acessibilidade no processo de busca com a utilização do software de síntese de voz NonVisual Desktop Access (NVDA - tradução livre: Acesso Não-Visual ao Ambiente de Trabalho).

O NVDA é um software livre, isto é, código aberto,de síntese de voz, ou leitor de tela, para sistemas Windows, idealizado por Michael Curran em 2006. Na atualidade é um dos softwares mais utilizados devido a sua disponibilidade a qualquer pessoa sem custos adicionais e de licença livre - o que permite a contribuição para a melhoria do software - e pelo fácil entendimento de seu design (ULIANA, 2008). O software é desenvolvido pela $N V$ Access com contribuições da comunidade mundial, e atualmente encontra-se na versão liberada no segundo semestre de 2015.

O desenvolvimento deste estudo justificou-se pela escassez de pesquisas realizadas para atender às necessidades informacionais de pessoas com deficiência visual que utilizam a internet, e a ferramenta web, como principais instrumentos de acesso à informação. A escolha pelo tema e sua contribuição para a ciência da informação se dão, principalmente, no que tange a atender com precisão às necessidades de informação desse grupo específico de usuários, e na responsabilidade social que deve ser absorvida pela ciência da informação, uma vez que faz parte de sua essência fornecer informação para quem necessita.

\section{2 elementos determinantes para o Processo de busca, NAVEGAÇÃO E RECUPERAÇÃO DA INFORMAÇÃO EM AMBIENTE WEB}

\begin{tabular}{l|l|l|l|l|l}
\hline (C) RDBCI: Rev. Digit. Bibliotecon. Cienc. Inf. & Campinas, SP & v.15 & n.1 & p. 211-233 & jan./abr. 2017 \\
\hline
\end{tabular}


A recuperação da informação tem como proposição a solução da problemática do excedente informacional, na qual áreas como a ciência da informação procuram propor soluções, de modo a possibilitar o sucesso no processo de busca e recuperação da informação (SARACEVIC, 1996; RODRIGUES et al., 2011). Para Saracevic (1996), a recuperação da informação compreende um dos agentes patrocinadores do desenvolvimento das bases científicas e tecnológicas, com influência na evolução da indústria informacional.

Entende-se nesta pesquisa que a recuperação da informação abrange o processo de busca da informação e a organização dos caminhos percorridos pelo usuário, em particular, ocorrido em rede de computadores e com a utilização da web. A internet é um meio de fácil acesso à informação, pois permite o armazenamento e o rápido compartilhamento da informação. Porém, há a problemática do excesso informacional nesse meio que tende a dificultar, de certa forma, a recuperação da informação.

Para que o usuário possa realizar uma busca com efetividade, o ideal é que seja o mais específico possível, podendo inclusive fazer uso de operadores booleanos para auxiliar no detalhamento de sua necessidade, pois quanto mais específico, maior a probabilidade de encontrar a informação desejada dentro da complexidade informacional que se tornou a web. Cabe ao usuário especificar sua necessidade. A tecnologia pode no máximo dar sugestões, muitas vezes baseadas em inteligência artificial, porém o sucesso da busca depende intrinsecamente do modo como é feita a pesquisa, ou seja, se o usuário não souber descrever corretamente a informação desejada, terá dificuldade em sua busca.

À tecnologia cabe auxiliar o usuário para que, de acordo com sua capacidade física e cognitiva, possa encontrar a informação desejada. Para contribuir com esse processo a arquitetura da informação pode ser um fator determinante. A arquitetura da informação fundamenta-se no princípio de que "é o resultado da distância cada vez maior entre o que compreendemos e o que achamos que deveríamos compreender. É o buraco negro que existe entre dados e conhecimento, e ocorre quando a informação não diz o que queremos ou precisamos saber" (WURMAN, 1991, p. 38).

De acordo como Morville e Rosenfeld (2006), a arquitetura da informação na web refere-se ao design estrutural de ambientes de informação compartilhados; a combinação dos esquemas de organização, rotulação, busca e navegação dentro de sites e intranets; a arte e a ciência de dar forma a produtos e experiências de informação para suportar a usabilidade e a encontrabilidade ou recuperabilidade; a disciplina emergente e uma comunidade de prática focada em trazer princípios de design e arquitetura no espaço digital.

A arquitetura da informação visa organizar os padrões inerentes dos dados e criar a estrutura ou mapa da informação de forma a permitir que outros encontrem seus próprios caminhos para o conhecimento tornando o complexo claro (WURMAN, 1997). Atualmente a arquitetura da informação emerge como uma disciplina preocupada com o design dos sites. Nesse contexto, para Nielsen (2000, p. 15), o objetivo da arquitetura da informação deve ser

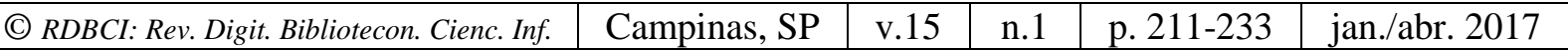


o de estruturar o site "para espelhar as tarefas dos usuários e suas visões do espaço de informação".

Para atingir esse objetivo Morville e Rosenfeld (2006) descreveram a arquitetura da informação de sites em quatro sistemas interdependentes: sistema de organização - que pode ser definido como um agrupamento e a categorização de todo o conteúdo informacional; sistema de navegação - especifica as maneiras de navegar, de se mover pelo espaço informacional e hipertextual; sistema de rotulação - estabelece as formas de representação e apresentação da informação definindo signos para cada elemento informativo; e o sistema de busca - determina as perguntas que o usuário pode fazer e o conjunto de respostas que irá obter. Cabe à arquitetura da informação projetar a organização, rotulação, navegação e busca nas páginas web, tendo sempre como foco o usuário.

A arquitetura da informação, na prática, procura balancear as particularidades e necessidades dos usuários, conteúdo e contexto, onde os seus componentes (organização, navegação, rotulação e busca) determinam a simplificação e dimensão das tarefas da arquitetura da informação que podem revelar a qualidade da organização dos elementos que possibilitam o processo de navegação dos usuários (MORVILLE; ROSENFELD, 2006). Entendendo-se que o conhecimento do usuário nesse processo é essencial para a efetiva disponibilização do conteúdo informacional em ambiente web. No caso de pessoas com deficiência visual, o procedimento de acessar a informação na web, ocorre segundo a acessibilidade promovida pelos componentes da arquitetura da informação.

Nessa perspectiva, referindo-se à acessibilidade na web para Dias (2003, p. 28), "se um sistema é fácil de usar, fácil de aprender e mesmo agradável ao usuário, mas não consegue atender a objetivos específicos de usuários específicos, ele não será usado, mesmo que seja oferecido gratuitamente". A autora esclarece ainda que: "acessibilidade é a capacidade de um produto ser flexível o suficiente para atender às necessidades e preferências do maior número possível de pessoas, além de ser compatível com tecnologias assistivas usadas por pessoas com necessidades especiais" (DIAS, 2003, p. 103). Nesse sentido, a acessibilidade é imprescindível para que haja facilidade de uso e estruturação de sites inclusivos para pessoas que possuam, ou não, algum tipo de limitação, como no caso deste estudo, pessoas com deficiência.

\section{ACESSO À INFORMAÇÃO E A PESSOA COM DEFICIÊNCIA VISUAL}

$\mathrm{Na}$ atualidade, com o aperfeiçoamento das tecnologias de informação e comunicação, novas possibilidades estão surgindo com a tecnologia digital, com relação à acessibilidade ao acesso à informação, permitindo que pessoas com deficiência visual adquiram informação não mais somente por impressos em braille, escrita ampliada ou por outros meios da tecnologia analógica (GOLUB, 2002). Contudo, de acordo com Nascimento (2006) e Borges (2009), na realidade da sociedade de países menos favorecidos, os aspectos das atividades relacionais das necessidades, buscas e usos da informação pela pessoa com deficiência visual,

\begin{tabular}{|c|c|c|c|c|c|}
\hline (C) RDBCI: Rev. Digit. Bibliotecon. Cienc. Inf. & Campinas, SP & v. 15 & n. & p. 211-233 & $\mathrm{ian} / \mathrm{abr} 2017$ \\
\hline
\end{tabular}


ainda se caracterizam por processos lentos, e, em determinados casos, inacessíveis, devido aos diversos fatores que as condicionam. As problemáticas que justificam as dificuldades na acessibilidade são diversas, principalmente, no que tange ao desconhecimento quanto às condições dessas pessoas, no tocante às suas necessidades informacionais, no processo de como realizam as suas buscas pela informação, as estratégias e suportes os quais fazem uso.

As diversas fontes de informação que na atualidade estão permitindo suprir as necessidades de informação - como livros, materiais de referência, publicações periódicas; aos meios eletrônicos, como banco de dados, publicações eletrônicas e a rede mundial de computadores - são ainda em sua maioria acessadas por meio da visão. A capacidade da visão é o sentido que mais transmite informações às pessoas por se relacionar com as diversas atividades da vida cotidiana, e, por isso, pessoas com deficiência visual precisam procurar meios alternativos para promoção da acessibilidade, de modo que a falta da visão não represente limitações ao estritamente necessário (YATACO MARÍN, 2009; WEI; LIRONG; CHUNMING, 2012).

Segundo Borges (2009), por motivos que dificultam a produção de livros em braille, as pessoas com cegueira, no contexto atual, não apenas procuram adquirir conhecimento por meio de informações impressas nesse sistema, mas buscam a utilização de outros formatos como o áudio e o digital. De acordo com Yataco Marín (2009), as atividades cotidianas de leituras de livros, revistas e páginas da web, não seriam possíveis para essas pessoas, se não houvesse os meios alternativos (originais para pessoas com deficiência visual) para o acesso à leitura. Atualmente, pessoas com cegueira podem acessar informações por tecnologias distintas, como as denominadas tecnologias assistivas.

Bersch (2008) e Melo, Costa e Soares (2006) afirmam que as tecnologias assistivas são recursos e serviços que têm como objetivo facilitar o desenvolvimento de atividades da vida cotidiana por pessoas com deficiência, auxiliando na locomoção, acessibilidade ao acesso à informação e comunicação, controle do ambiente, em diversas atividades diárias de estudo, trabalho e lazer (exemplos de tecnologias assistivas: cadeiras de rodas, bengalas, órteses e próteses, lupas, aparelhos auditivos, controles remotos, entre outros). As tecnologias assistivas são adaptadas às necessidades e características de cada pessoa, possibilitando o uso de recursos utilizados para proporcionar e facilitar o desenvolvimento das habilidades, de modo a aumentar as capacidades funcionais e promoção de autonomia. Concernente ao acesso à informação, a tecnologia assistiva compreende a adaptação e acessibilidade de tecnologias de informação e comunicação para pessoas com diversas limitações.

Os recursos para o acesso à informação pela pessoa com deficiência visual podem ser apresentados em dois aspectos: recursos que possibilitam o acesso à informação impressa e recursos que permitem o acesso à informação em meio digital. De acordo com os objetivos desta pesquisa, apenas serão evidenciados os recursos para o acesso à informação em meio digital, precisamente em ambiente web. 


\subsection{Recursos para acessibilidade na web utilizados por pessoas com cegueira}

De acordo com Craven e Brophy (2003), para a busca da informação em meio digital, como em sites, as pessoas com cegueira utilizam como principal recurso, os softwares de síntese de voz (Quadro 1) que possibilitam o acesso aos conteúdos informacionais digitais.

Quadro 1. Softwares de síntese de voz mais utilizados

\begin{tabular}{|c|c|c|}
\hline $\begin{array}{c}\text { SISTEMA DE } \\
\text { SÍNTESE DE VOZ }\end{array}$ & INSTITUIÇÃO/EMPRESA & ESPECIFICIDADES \\
\hline $\begin{array}{l}\text { DOSVOX/LINVOX } \\
\text { (Sistema Operacional) }\end{array}$ & $\begin{array}{l}\text { Núcleo de Computação Eletrônica / } \\
\text { Universidade Federal do Rio de } \\
\text { Janeiro (NCE/UFRJ) } \\
\text { (http://intervox.nce.ufrj.br/dosvox/) }\end{array}$ & $\begin{array}{l}\text { Ambiente Windows (DOSVOX), ambiente } \\
\text { Linux (LINVOX) interfaces adaptativas, } \\
\text { editor de texto, recurso para impressão em } \\
\text { tinta e em braile, jogos didáticos e lúdicos, } \\
\text { calculadora, acesso a web, entre outros }\end{array}$ \\
\hline $\begin{array}{l}\text { NVDA }(\text { NON VISUAL } \\
\text { DESKTOP ACCESS })\end{array}$ & $\begin{array}{l}\text { Michael Curran / NVDA team } \\
\text { (http://www.nvaccess.org/) }\end{array}$ & $\begin{array}{l}\text { Ambiente Windows, usos de softwares, } \\
\text { navegação web, anúncio automático de } \\
\text { texto a partir do posicionamento do mouse, } \\
\text { podendo ser rodado diretamente de um } \\
\text { cabo USB ou qualquer mídia portátil, entre } \\
\text { outros }\end{array}$ \\
\hline ORCA & $\begin{array}{l}\text { Sun Microsystems / Empresa } \\
\text { internacional } \\
\text { (projects.gnome.org/orca/) }\end{array}$ & $\begin{array}{l}\text { Ambiente Linux, Solaris, BSD, e baseados } \\
\text { em Unix, combinações de síntese de fala, } \\
\text { braile, ampliação, openoffice, firefox, entre } \\
\text { outros }\end{array}$ \\
\hline DELTA TALK & $\begin{array}{l}\text { MicroPower / Empresa brasileira } \\
\text { (http://www.micropower.com.br/) }\end{array}$ & $\begin{array}{l}\text { Ambiente Windows, escolha por tipo de } \\
\text { voz, controle de velocidade de pronúncia e } \\
\text { tonalidade de voz, entre outros }\end{array}$ \\
\hline VIRTUAL VISION & $\begin{array}{l}\text { MicroPower / Empresa brasileira } \\
\text { (http://www.micropower.com.br/) }\end{array}$ & $\begin{array}{l}\text { Ambiente Windows, aplicativos office, } \\
\text { navegação web, usos de softwares de } \\
\text { comunicação, entre outros }\end{array}$ \\
\hline $\begin{array}{l}\text { JAWS (JOB ACCESS } \\
\text { WITH SPEECH) }\end{array}$ & $\begin{array}{l}\text { Freedom Scientific / Empresa } \\
\text { internacional } \\
\text { (http://www.freedomscientific.com/) }\end{array}$ & $\begin{array}{l}\text { Ambiente Windows, utilização de } \\
\text { softwares, navegação web, entre outros }\end{array}$ \\
\hline
\end{tabular}

Fonte: Dados de pesquisa (2015).

Considera-se, de modo geral, que o próprio computador somente pode ser utilizado pela pessoa com cegueira com a utilização desses softwares que permitem a obtenção da leitura imediata, por se tratarem de dispositivos capazes de converter textos digitais em textos falados (sintetizados). Os materiais de informação, adaptados por processos de digitalização com a finalidade de serem utilizados por pessoas com cegueira, são basicamente interpretados por esses softwares sem maiores problemas que comprometam a leitura do material. Entretanto, deve-se considerar algumas dificuldades encontradas por essas pessoas durante o processo de busca das informações disponibilizadas tanto em softwares e arquivos, como também a navegabilidade em sites.

As dificuldades apresentadas pelas pessoas com cegueira em relação ao acesso à informação em sites tratam-se das principais discussões no campo da acessibilidade na web. É importante considerar que os softwares de síntese de voz têm possibilitado inúmeros benefícios à acessibilidade na web. No entanto, esses softwares não são capazes de fazer a totalidade da leitura e, consequentemente, a busca e recuperação eficaz das informações

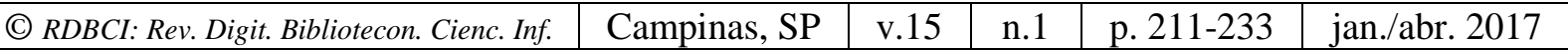


contidas nos sites e páginas, caso não se encontrem estruturados de forma acessível. Nesse caso, é necessário que haja o acompanhamento de especificações, durante o processo de desenvolvimento de sites, para que os softwares de síntese de voz possam realizar leitura prática, estratégica e rápida das informações para que a busca seja efetiva.

De acordo com Santos e Nascimento (2006), as principais problemáticas vivenciadas pelas pessoas com cegueira são as dificuldades em obter informações disponíveis em formato visual, interação com o uso de dispositivo diferente do teclado, disposição de links em documento, navegação pelos conceitos espaciais e a distinção entre sons por uma voz produzida por síntese. Santos e Nascimento (2006) e Melo e Baranauskas e Souza (2006) salientam que, com a observação dessas dificuldades, o World Wide Web Consortium (W3C), órgão responsável pela coordenação, elaboração e padronização das regras de acessibilidade a serem seguidas na construção de sites e páginas na web, pela Web Accessibility Initiative (WAI), desenvolveu o documento de recomendações Web Content Accessibility Guidelines (WCAG) para promoção da acessibilidade na web e especificações técnicas. Esse documento é referência em âmbito mundial, o qual atualmente encontra-se na versão 2.0, acompanhando o processo evolutivo da web.

Segundo Santos e Nascimento (2006), as aplicações das técnicas de acessibilidade propostas pelo WCAG, auxiliam não somente as pessoas com deficiência, mas também aos demais usuários, no tocante aos tipos de conexões e navegadores mais antigos, devido a essas técnicas permitirem o desenvolvimento de sites e páginas mais flexíveis e com maior acessibilidade, sabendo-se que a aplicação dessas recomendações não implica deixar de utilizar recursos como: imagens e vídeos, mas possibilitar que o desenvolvedor torne todo o conteúdo multimídia acessível a um público diversificado.

As recomendações de acessibilidade para conteúdo web constituem-se como princípios gerais que propõem a organização do conteúdo web de forma a torná-lo acessível aos usuários com deficiência. No entanto, deve-se considerar que:

Embora o foco esteja em promover a acessibilidade para pessoas com deficiência (perceptual, motora, cognitiva), a WAI reconhece que a observação de suas recomendações também pode ampliar o acesso ao conteúdo da web para pessoas em situações de acesso diversificadas, seja por utilizarem diferentes tecnologias em sua navegação (navegadores gráficos para ambiente desktop, navegadores textuais, sintetizadores de voz, ampliadores de telas, celulares), seja por operarem em condições restritas (ex. tecnologia obsoleta, ambiente com ou sem ruído, iluminação variada, mobiliário em configuração diferente da convencional). (MELO, 2007, p. 24).

A série de recomendações, ou princípios gerais, como evidenciada por Melo (2007), contida no documento WCAG, reúne pontos de verificação (checkpoints), que designam como as recomendações precisam ser compreendidas segundo a determinação da 
especificidade de uma dada situação-problema. Entende-se que com o desenvolvimento da $w e b$, as recomendações estabelecidas pelo W3C passam por reformulações para que a condição de acessibilidade seja contínua de acordo com as novas tecnologias empregadas. Assim sendo, nas recomendações estabelecidas em 1999, o W3C priorizou em 14 recomendações os temas que asseguravam a harmonia das páginas (que contemplavam desde as alternativas para conteúdo sonoro e visual, a indicação da utilização de tecnologias e recomendações do W3C) e a conteúdo compreensível e navegável (que evidenciava a oferta de informações de contexto, de orientação, e mecanismos de navegação claros, assim como a certificação da clareza e simplicidade dos documentos) (W3C, 1999; MELO, 2007).

O W3C procurou desenvolver novas recomendações para o conteúdo web em conformidade com as atuais tecnologias utilizadas e a novas situações comunicacionais vivenciadas, bem como de acordo com a diversificação do público personificado pelos desenvolvedores de políticas, gerentes, designers gráficos e editores (MELO, 2007; W3C, 2008). Segundo Melo (2007, p. 25), as novas recomendações agrupam-se com base em quatro "princípios de acessibilidade considerados fundamentais ao acesso e ao uso da web por qualquer pessoa"; sendo que "para cada recomendação são associados critérios de sucesso à sua aplicação, com o propósito de tornar as verificações mais precisas". Encontram-se organizados no Quadro 2, os princípios e as recomendações estabelecidos pelo W3C para a web 2.0.

QUADRO 2. Recomendações de acessibilidade para conteúdo web (web 2.0)

\begin{tabular}{|c|c|}
\hline PRINCÍPIOS & RECOMENDAÇÕES \\
\hline \multirow[t]{4}{*}{$\begin{array}{l}\text { Princípio 1: Perceptível - Informação e } \\
\text { componentes de interface de usuário devem } \\
\text { ser perceptíveis aos usuários }\end{array}$} & $\begin{array}{l}\text { Recomendação 1.1. Ofereça texto alternativo para todo conteúdo } \\
\text { não textual de maneira que possa ser modificado para outras } \\
\text { formas que as pessoas necessitem como impressão ampliada, } \\
\text { Braille, voz, símbolos ou linguagem mais simples. }\end{array}$ \\
\hline & $\begin{array}{l}\text { Recomendação 1.2. Ofereça alternativas sincronizadas ao } \\
\text { conteúdo multimídia. }\end{array}$ \\
\hline & $\begin{array}{l}\text { Recomendação 1.3. Crie conteúdo que possa ser apresentado em } \\
\text { diferentes maneiras (ex. lido em voz alta, layout mais simples) } \\
\text { sem perda da informação ou estrutura. }\end{array}$ \\
\hline & $\begin{array}{l}\text { Recomendação 1.4. Facilite para a pessoa com deficiência ver e } \\
\text { ouvir o conteúdo inclusive separar o que está em primeiro plano } \\
\text { do que está ao fundo. }\end{array}$ \\
\hline \multirow{4}{*}{$\begin{array}{l}\text { Princípio 2: Operável - Componentes de } \\
\text { interface no conteúdo devem ser operáveis } \\
\text { pelos usuários }\end{array}$} & $\begin{array}{l}\text { Recomendação } 2.1 \text {. Torne toda a funcionalidade disponível a } \\
\text { partir de um teclado. }\end{array}$ \\
\hline & $\begin{array}{l}\text { Recomendação 2.2. Ofereça aos usuários com deficiência tempo } \\
\text { suficiente para ler e usar o conteúdo. }\end{array}$ \\
\hline & $\begin{array}{l}\text { Recomendação 2.3. Não crie conteúdo que possa causar } \\
\text { convulsão. }\end{array}$ \\
\hline & $\begin{array}{l}\text { Recomendação 2.4. Ofereça maneiras para auxiliar os usuários } \\
\text { com deficiência a navegarem, a encontrarem conteúdo, e a } \\
\text { determinarem onde estão. }\end{array}$ \\
\hline \multirow{3}{*}{$\begin{array}{l}\text { Princípio 3: Compreensível - Informação e } \\
\text { operação da interface de usuário devem ser } \\
\text { compreensíveis pelos usuários }\end{array}$} & $\begin{array}{l}\text { Recomendação 3.1. Faça o conteúdo do texto legível e } \\
\text { compreensível. }\end{array}$ \\
\hline & $\begin{array}{l}\text { Recomendação 3.2. Faça as páginas web aparecerem e operarem } \\
\text { de maneira previsível. }\end{array}$ \\
\hline & $\begin{array}{l}\text { Recomendação 3.3. Ajude os usuários a evitarem e corrigirem } \\
\text { erros. }\end{array}$ \\
\hline Princípio 4: Robusto - O conteúdo deve ser & Recomendação 4.1. Maximize a compatibilidade com agentes de \\
\hline
\end{tabular}


"robusto" o suficiente para ser interpretado $\quad$ usuários atuais e futuros, incluindo tecnologias assistivas. de maneira confiável por uma grande

variedade de agentes de usuários, incluindo

tecnologias assistivas

Fonte: MELO (2007, p. 25-26, quadro adaptado); W3C (2008).

Os princípios de acessibilidade estabelecidos para web 2.0 compreendem quatro pilares para a efetividade do processo de acessibilidade em ambiente web, conforme as bases da perceptibilidade, operabilidade, compreensibilidade e compatibilidade.

Pela perceptibilidade, no sentido de prover mecanismos e recursos tais que os usuários, em suas diferentes capacidades perceptuais (visual, auditiva, etc), sejam capazes de perceber seu conteúdo. Operabilidade é um segundo pilar, que garante não apenas o acesso, mas principalmente a interação com sistemas na web. O terceiro diz respeito à compreensibilidade, que garante não apenas o acesso e a interação, mas a inteligibilidade dos elementos de tal forma a possibilitar o uso. Finalmente, a compatibilidade do conteúdo é necessária para possibilitar o acesso, a interação e o uso com múltiplos agentes de usuário e artefatos. (MELO, 2007, p. 26).

Com a utilização de softwares de síntese de voz como a ferramenta responsável pela navegabilidade na web, e consequentemente, pelo processo de busca e recuperação da informação, deve-se considerar os aspectos das recomendações para acessibilidade, sabendose que as possibilidades evidenciadas pelos conteúdos web, e as limitações existentes nos processos de busca e recuperação por falta de padronização na construção de sites e páginas, compreendem determinações e desconhecimento quanto às necessidades dessas pessoas, 0 que acabam por limitar a acessibilidade ao conteúdo informacional produzido e compartilhado, diante disso, constata-se que ainda são muitos os entraves existentes que impedem que a acessibilidade se torne uma condição de acesso universal efetivo (CHRISTENSEN; STEVNS, 2012).

\section{ASPECtos da PeSquisa desenvolvida e ANÁlise dos dados OBTIDOS}

A pesquisa desenvolvida fundamentou-se nos estudos dos processos de busca, navegação e recuperação da informação em ambiente web centrados no usuário, de modo a estabelecer o desenvolvimento de um checklist simples, que pode ser utilizado em avaliações de processos de busca, navegação e recuperação da informação pelas pessoas com cegueira. A pesquisa para a construção do checklist estabeleceu-se a partir da utilização do método de simulação.

A pesquisa se configurou como exploratória e descritiva, por ter se centrado no conhecimento do processo de busca, navegação e recuperação de informação, com foco nas técnicas e estratégias utilizadas por pessoas com cegueira, bem como na apresentação desse

\begin{tabular}{|c|c|c|c|c|c|}
\hline (C) RDBCI: Rev. Digit. Bibliotecon. Cienc. Inf. & Campinas, SP & v. 15 & n. & n. 211-233 & jan./abr. 2017 \\
\hline
\end{tabular}


processo de forma a especificar suas características e propriedades intrínsecas (HERNÁNDEZ SAMPIERI; HERNÁNDEZ COLLADO; BAPTISTA LUCIO, 2006). A natureza determinou-se como qualitativa, devido à necessidade de se apresentar os resultados da coleta de dados, adotada por esta pesquisa, em dados qualitativos perante a observação realizada conforme a simulação estabelecida para a construção dos campos de questionamento do checklist (ROGERS; SHARP; PREECE, 2013).

Como metodologia de investigação, caracterizou-se como estudo de caso, pelo fato desta pesquisa se focalizar na investigação de caso específico, delimitado e contextualizado, de modo a permitir a realização de busca circunstanciada de informações sobre o caso, procurando conhecer esse processo em seus aspectos característicos, bem como pelo fato do estudo de caso ser de grande utilidade em pesquisas exploratórias (VENTURA, 2007).

A coleta de dados determinada por este estudo tornou-se fundamental para evidenciar o estabelecimento de requisitos e avaliações. No tocante aos requisitos, a coleta de dados visa à obtenção de dados que sejam suficientes, precisos e relevantes para produção de conjunto de requisitos estáveis. Para a área da avaliação, o procedimento de coleta de dados possibilita a captação de reações e comportamento de usuários com relação ao seu desempenho com sistemas e protótipos (ROGERS; SHARP; PREECE, 2013).

O procedimento de coleta de dados se estabeleceu com a utilização do método de aplicação e verificação direta, em simulação ocorrida em ambiente parcialmente controlado laboratório de tecnologias assistivas voltado para usuários com deficiência visual e dislexia -, com a utilização de software de síntese de voz em procedimentos de simulação de busca, navegação e recuperação de informação, com base em técnicas e procedimentos utilizados por pessoas com cegueira conduzidas pelos autores desta pesquisa, sem a participação dessas pessoas como usuárias do sistema em processo de busca.

Esclarece-se que para o desenvolvimento do checklist proposto por esta pesquisa, apenas foi considerada a identificação dos pontos de critérios para acessibilidade, para que tornasse possível a construção da estrutura básica de um checklist simples que poderá ser utilizado como modelo para a construção de checklists mais gerais e específicos de acessibilidade a serem desenvolvidos em conjunto com usuários com cegueira em processo de navegação web. Condição essa que centralizou esta pesquisa na aplicação e comportamento técnico do sistema de síntese de voz em processo de busca web, não necessitando da presença do real usuário do sistema (pessoa com deficiência visual).

Para o método de aplicação, o uso do instrumento de verificação direta possibilitou a supervisão da simulação no momento de seu desenvolvimento, permitindo a observação durante a aplicação e procedimentos desenvolvidos em processo de verificação contínua.

Para a construção do checklist, além do desenvolvimento da simulação, necessitou-se, também, ter como base as reformulações de quesitos de guias referentes à avaliação de acessibilidade em sistemas, conteúdos web, ergonomia, entre outros, para a efetivação de uma \begin{tabular}{l|l|l|l|l|l} 
(C) RDBCI: Rev. Digit. Bibliotecon. Cienc. Inf. & Campinas, SP & v.15 & n.1 & p. 211-233 & jan./abr. 2017 \\
\hline
\end{tabular} 
proposta que possibilite verificações em processos de busca, navegação e recuperação da informação. A escolha pelo ambiente parcialmente controlado para a prática da simulação, laboratório especializado em tecnologias assistivas, deu-se pela necessidade de se utilizar os hardwares e softwares costumeiramente utilizados por pessoas com cegueira, de modo que a pesquisa se estabeleceu em plano mais fiel possível, com a simulação das necessidades de informação dessas pessoas, como, por exemplo, o conhecimento dos comandos (atalhos) utilizados para o software de síntese de voz. Tratou-se de uma forma de estruturar o ambiente para simular o contexto da pessoa com cegueira.

A simulação desenvolvida, sem a participação do usuário cego, não implicou em obtenção de dados não confiáveis, pois os testes propostos por este estudo centraram-se nos aspectos dos processos de busca, navegação e recuperação em ambiente web apenas considerando a navegação básica a partir da aplicação do sistema de síntese de voz, na qual muitos dos atalhos são comuns entre os softwares de síntese de voz mais utilizados (Quadro 1). Nesse processo é fundamental considerar também o navegador, buscador e o sistema operacional utilizados na pesquisa: navegador Firefox versão 33.0.1, buscador Google e sistema operacional Windows 7.

\subsection{Encaminhamento da simulação e apresentação do checklist}

A simulação foi estruturada de modo a permitir o desenvolvimento de ambiente favorável, que possibilitou a aplicação e verificação a partir da utilização do NVDA, estabelecendo procedimentos de busca, navegação e recuperação da informação, de forma a analisar aspectos de acessibilidade nesse processo. Para a construção do checklist, necessitouse estabelecer três etapas: a Etapa I correspondeu à identificação dos critérios para acessibilidade; a Etapa II analisou, a partir dos critérios, a relação dos princípios da recomendação W3C; e a Etapa III considerou o desenvolvimento da simulação com o NVDA, a partir dos resultados obtidos nas etapas anteriores.

Para a simulação, estabeleceu-se a seleção dos termos de pesquisa. Os termos sugeridos pela pesquisa foram: 1. Musicografia Braille; 2. Eleições 2014. Optou-se por esses termos devido a dois determinantes: o fato de serem compostos, o que permite resultados mais específicos, centralizando dessa forma o processo de busca; e por corresponderem a áreas diferenciadas: o termo "Musicografia Braille" refere-se a uma área específica do universo do acesso à informação musical para pessoas com cegueira, o que trouxe resultados de busca mais focalizados e, consequentemente, sites possivelmente mais acessíveis; o termo "Eleições 2014", compreende um termo mais generalizado que proporcionou o retorno de resultados de sites diversificados, acessíveis e não acessíveis. Esse procedimento possibilitou a construção do checklist com base em um comparativo das duas dimensões "acessível" e "não acessível". Quanto à análise dos resultados obtidos, para cada termo, com a busca, centrou-se na primeira página de resultados recuperados pelo buscador Google, evidenciando o estudo dos primeiros seis sites. 
Na Etapa I, com base no estudo de Sales (2002), que tem como foco a melhoria da acessibilidade da web visando usuários idosos; em análises no documento da recomendação W3C para web 2.0; e nos estudos de Downey e Banerjee (2010), foi possível desenvolver, a partir de quatro critérios selecionados para acessibilidade, 17 questões que visaram proporcionar, para este estudo, a verificação de acesso à informação. Apresentam-se no Quadro 3, os critérios selecionados.

QUADRO 3. Critérios para acessibilidade

\begin{tabular}{|l|l|}
\hline \multicolumn{1}{|c|}{ CRITÉRIOS } & \multicolumn{1}{c|}{ MOTIVO PARA SELEÇÃO } \\
\hline Profundidade & $\begin{array}{l}\text { Detalhamento específico do processo de busca a partir da possível interação do usuário- } \\
\text { software de síntese de voz com o sistema de busca. }\end{array}$ \\
\hline Amplitude & $\begin{array}{l}\text { Verificação geral do processo de busca com foco no processo de navegação e } \\
\text { recuperação da informação. }\end{array}$ \\
\hline Tempo & $\begin{array}{l}\text { Tempo decorrido pelo software de síntese de voz e usuário durante o processo de busca } \\
\text { e navegaçãoaté a recuperação da informação desejada. }\end{array}$ \\
\hline Satisfação & $\begin{array}{l}\text { Possível satisfação do usuário quanto ao processo de busca acerca da navegação e } \\
\text { recuperação da informação desejada. }\end{array}$ \\
\hline
\end{tabular}

Fonte: Elaborado pelos autores (2015).

Com análise dos critérios a partir da avaliação dos níveis (Nível 1 e Nível 2), na Etapa II, verificou-se a importância da identificação dos princípios (Quadro 4) considerados pelas recomendações para acessibilidade web, concernente a percepção, operacionalização, compreensão e autenticação.

QUADRO 4. Princípios que subsidiaram os critérios para acessibilidade

\begin{tabular}{|l|l|}
\hline \multicolumn{1}{|c|}{ PRINCÍPIOS } & \multicolumn{1}{c|}{ RECOMENDAÇÕES } \\
\hline Percepção & $\begin{array}{l}\text { No processo de ordem de navegação, verificação de textos alternativos ao } \\
\text { conteúdo não textual, alternativas ao conteúdo e suas mídias, layout simples (sem } \\
\text { perda da informação ou estrutura), separação do conteúdo de primeiro e segundo } \\
\text { planos. }\end{array}$ \\
\hline Operacionalização & $\begin{array}{l}\text { Disponibilidade a partir do teclado, tempo de leitura e uso do conteúdo, conteúdo } \\
\text { confundível, auxílio a navegabilidade para o encontro ao conteúdo. }\end{array}$ \\
\hline Compreensão & $\begin{array}{l}\text { Legibilidade textual, previsibilidade da operacionalização das páginas, } \\
\text { entendimento dos erros. }\end{array}$ \\
\hline Autenticação & Compatibilidade entre usuários e tecnologias assistivas. \\
\hline
\end{tabular}

Fonte: Elaborado pelos autores (2015).

A determinação desses quesitos obtidos, a partir dos critérios para acessibilidade, foi evidenciada conforme o estabelecimento das aplicações e verificações efetivadas ao longo da simulação de busca e navegação, onde foram consideradas as principais condições exigidas para a acessibilidade tanto da pessoa com cegueira quanto do próprio software de síntese de voz. Com os resultados obtidos com a simulação, especificaram-se no checklist as possíveis alternativas de profundidade, amplitude, tempo e satisfação.

No Quadro 5 é apresentada a estruturação do checklist conforme o resultado da simulação ocorrida na Etapa III, mostrando os possíveis aspectos inerentes ao processo de busca, navegação e recuperação da informação com a utilização do NVDA. Esses aspectos

\begin{tabular}{|c|c|c|c|c|c|}
\hline (C) RDBCI: Rev. Digit. Bibliotecon. Cienc. Inf. & Campinas, SP & v. 15 & n. & p. 211-233 & jan/abr. 2017 \\
\hline
\end{tabular}


consideraram as características da busca em que se aplicaram as questões e o tratamento que as originaram; que no caso deste estudo, referiu-se à simulação.

QUADRO 5. Checklist para verificação de acessibilidade no processo de busca, navegação e recuperação da informação

\begin{tabular}{|c|c|}
\hline \multirow{2}{*}{\multicolumn{2}{|c|}{ 1. PROFUNDIDADE }} \\
\hline & \\
\hline \multirow[t]{4}{*}{ Operacionalização } & $\begin{array}{l}\text { Como se estabelece a navegação na página inicial do buscador na identificação da } \\
\text { caixa de combinação editável? }\end{array}$ \\
\hline & Aplica-se: Função de busca direta. \\
\hline & $\begin{array}{l}\text { Possíveis alternativas identificadas: } \\
\text { a. Automaticamente; } \\
\text { b. Navegação por elementos da página inicial do buscador. }\end{array}$ \\
\hline & Origem da questão: Estudo estabelecido a partir da simulação. \\
\hline \multicolumn{2}{|c|}{ Nível 1 (Página dos resultados do sistema de busca) } \\
\hline \multirow[t]{4}{*}{$\begin{array}{l}\text { Percepção e } \\
\text { Operacionalização }\end{array}$} & $\begin{array}{l}\text { Como se estabelece a navegação pelos elementos na página dos resultados; Dentro } \\
\text { da página (Nível 1), aprofundou-se até qual item de resultado? }\end{array}$ \\
\hline & Aplica-se: Função de busca direta. \\
\hline & $\begin{array}{l}\text { Possíveis alternativas identificadas } \\
\text { Elementos focalizáveis; Navegação detalhada; Não se aplica }\end{array}$ \\
\hline & Origem da questão: Estudo estabelecido a partir da simulação. \\
\hline \multirow[t]{4}{*}{$\begin{array}{l}\text { Percepção e } \\
\text { Operacionalização }\end{array}$} & $\begin{array}{l}\text { A busca estabelecida segue por resultado obtido ou pela leitura de mais de um } \\
\text { resultado? A busca é feita na ordem horizontal, ou seja, por ordem de resultado } \\
\text { obtido; ou a busca é feita na ordem vertical, ou seja, desce no resultado obtido para } \\
\text { só então adentrar na página que se considera ter a informação? }\end{array}$ \\
\hline & Aplica-se: Função de busca direta. \\
\hline & $\begin{array}{l}\text { Possíveis alternativas identificadas: } \\
\text { a. Por resultado; } \\
\text { b. Por mais de um resultado. }\end{array}$ \\
\hline & Origem da questão: Estudo estabelecido a partir da simulação. \\
\hline \multicolumn{2}{|c|}{ Nível 2 (Navegação dentro dos sites) } \\
\hline \multirow[t]{4}{*}{ Percepção } & $\begin{array}{l}\text { A busca estabelecida no site é desenvolvida por leitura detalhada da página? Dentro } \\
\text { das páginas, nível } 2 \text {, o usuário a detalha ou para logo no início? }\end{array}$ \\
\hline & Aplica-se: Função de busca direta. \\
\hline & $\begin{array}{l}\text { Possíveis alternativas identificadas } \\
\text { Leitura completa; Leitura incompleta. }\end{array}$ \\
\hline & Origem da questão: Estudo estabelecido a partir da simulação. \\
\hline \multirow[t]{4}{*}{ Percepção } & $\begin{array}{l}\text { Existência de textos alternativos ao conteúdo não textual (imagens, gráficos, entre } \\
\text { outros)? Com a existência de várias mídias, são de fácil acesso ou existe alternativa } \\
\text { de conteúdo? }\end{array}$ \\
\hline & Aplica-se: Função de arquitetura da informação. \\
\hline & Não houve a identificação de alternativas \\
\hline & $\begin{array}{l}\text { Origem da questão: Recomendações de acessibilidade para conteúdo web (WCAG } \\
\text { 2.0/W3C) com verificação de estudo a partir da simulação. }\end{array}$ \\
\hline \multirow[t]{4}{*}{ Percepção } & $\begin{array}{l}\text { De acordo com a estrutura (layout), são de fácil assimilação ou ocorre perda de } \\
\text { informação? }\end{array}$ \\
\hline & Aplica-se: Função de arquitetura da informação. \\
\hline & Não houve identificação de alternativas. \\
\hline & $\begin{array}{l}\text { Origem da questão: Recomendações de acessibilidade para conteúdo web (WCAG } \\
\text { 2.0/W3C) com verificação de estudo a partir da simulação. }\end{array}$ \\
\hline \multirow[t]{4}{*}{ Percepção } & É fácil a identificação do conteúdo de primeiro plano e segundo plano? \\
\hline & Aplica-se: Função de arquitetura da informação. \\
\hline & Não houve identificação de alternativas. \\
\hline & $\begin{array}{l}\text { Origem da questão: Recomendações de acessibilidade para conteúdo web (WCAG } \\
\text { 2.0/W3C) com verificação de estudo a partir da simulação. }\end{array}$ \\
\hline Operacionalização & Dificuldades encontradas com a utilização dos atalhos (disponibilidade a partir do \\
\hline
\end{tabular}

\begin{tabular}{|l|l|l|l|l|l|} 
(C) RDBCI: Rev. Digit. Bibliotecon. Cienc. Inf. & Campinas, SP & v.15 & n.1 & p. 211-233 & jan./abr. 2017 \\
\hline
\end{tabular} 


\begin{tabular}{|c|c|}
\hline & teclado)? \\
\hline & Aplica-se: Função de arquitetura da informação. \\
\hline & Não houve identificação de alternativas. \\
\hline & $\begin{array}{l}\text { Origem da questão: Recomendações de acessibilidade para conteúdo web (WCAG } \\
\text { 2.0/W3C) com verificação de estudo a partir da simulação. }\end{array}$ \\
\hline Operacionalização & Como se procede o tempo de leitura e uso do conteúdo? \\
\hline & Aplica-se: Função de arquitetura da informação. \\
\hline & Não houve identificação de alternativas. \\
\hline & $\begin{array}{l}\text { Origem da questão: Recomendações de acessibilidade para conteúdo web (WCAG } \\
\text { 2.0/W3C) com verificação de estudo a partir da simulação. }\end{array}$ \\
\hline Operacionalização & $\begin{array}{l}\text { Identifica-se a existência de confusão de conteúdo? Existência de auxílio no } \\
\text { processo de navegação para o encontro do conteúdo desejável? }\end{array}$ \\
\hline & Aplica-se: Função de arquitetura da informação. \\
\hline & Não houve identificação de alternativas. \\
\hline & $\begin{array}{l}\text { Origem da questão: Recomendações de acessibilidade para conteúdo web (WCAG } \\
\text { 2.0/W3C) com verificação de estudo a partir da simulação. }\end{array}$ \\
\hline Compreensão & $\begin{array}{l}\text { Quanto aos textos, identifica-se a sua legibilidade, a operacionalização da página e } \\
\text { os entendimentos dos possíveis erros? }\end{array}$ \\
\hline & Aplica-se: Função de arquitetura da informação. \\
\hline & Não houve identificação de alternativas. \\
\hline & $\begin{array}{l}\text { Origem da questão: Recomendações de acessibilidade para conteúdo web (WCAG } \\
\text { 2.0/W3C) com verificação de estudo a partir da simulação. }\end{array}$ \\
\hline 2. AMPLITUDE & \\
\hline Nível 2 (Navegação & o dos sites) \\
\hline Percepção & $\begin{array}{l}\text { A recuperação da informação foi efetivamente estabelecida no site? Encontrou-se o } \\
\text { que queria? }\end{array}$ \\
\hline & Aplica-se: Função de recuperação da informação. \\
\hline & $\begin{array}{l}\text { Possíveis alternativas identificadas: } \\
\text { a. Informação completa; } \\
\text { b. Informação incompleta. }\end{array}$ \\
\hline & Origem da questão: Estudo estabelecido a partir da simulação. \\
\hline Nível 2 (Navegação & o dos sites) \\
\hline Compreensão & $\begin{array}{l}\text { Há ocorrência de desistência no processo de busca em sites escolhidos? (Há } \\
\text { desistência? Se sim, ocorreu em que nível: 1-na página com o resultado da busca } \\
\text { com o sistema de buscador ou 2-dentro de um dos sites? Qual tempo decorrido até a } \\
\text { desistência?) }\end{array}$ \\
\hline & Aplica-se: Função de busca direta. \\
\hline & $\begin{array}{l}\text { Possíveis alternativas identificadas: } \\
\text { a. Desistência; } \\
\text { b. Não desistência. }\end{array}$ \\
\hline & Origem da questão: Estudo estabelecido a partir da simulação. \\
\hline $\begin{array}{l}\text { 3. TEMPO (BUSC } \\
\text { [RETORNO SONO }\end{array}$ & ISTEMA], PESQUISA [USUÁRIO], SOFTWARE DE SÍNTESE DE VOZ \\
\hline Operacionalização & $\begin{array}{l}\text { O tempo de retorno da busca efetuada pelo sistema (buscadores) (Em quanto tempo } \\
\text { os buscadores retornaram os resultados?) }\end{array}$ \\
\hline & Aplica-se: Tempo de Busca pelo Sistema (TBS). \\
\hline & $\begin{array}{l}\text { Possíveis alternativas identificadas: } \\
\text { a. } \quad \text { TBS }>0,70 \mathrm{seg} ; \\
\text { b. } \quad \text { TBS }<0,70 \mathrm{seg} ; \\
\text { c. } \quad \text { TBS }=0,70 \mathrm{seg}\end{array}$ \\
\hline & Origem da questão: Estudo estabelecido a partir da simulação. \\
\hline Operacionalização & $\begin{array}{l}\text { O tempo estabelecido pelo usuário no processo de pesquisa (processo que se inicia } \\
\text { com a busca, verifica-se com a navegação e termina com a recuperação da } \\
\text { informação considerada satisfatória) (Quanto tempo desde a busca até a efetiva } \\
\text { recuperação da informação?) }\end{array}$ \\
\hline & Aplica-se: Tempo de Pesquisa pelo Usuário (TPU). \\
\hline & $\begin{array}{l}\text { Possíveis alternativas identificadas: } \\
\text { a. TPU> 50min; } \\
\text { b. TPU<50min; } \\
\end{array}$ \\
\hline
\end{tabular}

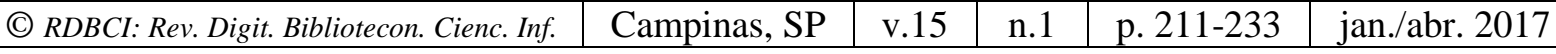




\begin{tabular}{|c|c|}
\hline & c. $\mathrm{TPU}=50$. \\
\hline & Origem da questão: Estudo estabelecido a partir da simulação. \\
\hline Operacionalização & $\begin{array}{l}\text { O tempo de retorno sonoro do software de síntese de voz para o início da busca pelo } \\
\text { usuário (Em quanto tempo o buscador retorna o resultado com o software de síntese } \\
\text { de voz?) }\end{array}$ \\
\hline & Aplica-se: Tempo de Retorno Sonoro (TRS). \\
\hline & $\begin{array}{l}\text { Possíveis alternativas identificadas: } \\
\text { a. } \quad \text { TRS }>2 \mathrm{seg} \\
\text { b. } \quad \text { TRS }<2 \mathrm{seg} \\
\text { c. } \quad \text { TRS }=2 \mathrm{seg}\end{array}$ \\
\hline & Origem da questão: Estudo estabelecido a partir da simulação. \\
\hline 4. SATISFAÇÃO (I & SSIDADES SATISFEITAS) \\
\hline Percepção & $\begin{array}{l}\text { Verifique se o processo de busca estabelecida possibilitou ao usuário satisfazer a } \\
\text { necessidade de informação (Conseguiu-se a efetiva recuperação da informação?) }\end{array}$ \\
\hline & Aplica-se: Informação (obtenção). \\
\hline & $\begin{array}{l}\text { Possíveis alternativas identificadas: } \\
\text { a. Satisfação completa; } \\
\text { b. Satisfação incompleta. }\end{array}$ \\
\hline & Origem da questão: Estudo estabelecido a partir da simulação. \\
\hline
\end{tabular}

Fonte: Elaborados pelos autores (2015).

Com o critério de Profundidade, tornou-se possível determinar o processo de navegação com o sistema de busca para o procedimento de navegabilidade, aprofundamento dentro da página de resultados, navegação segundo a ordem (horizontal e vertical) dos resultados, e o detalhamento da busca e navegação conforme os Níveis 1 e 2. Nessa parte, foram constatados aspectos que contemplaram os campos da Percepção, Operacionalização e Compreensão, evidenciados com a utilização do sistema de busca, e nos Níveis 1 (referente a página de resultados do sistema de busca) e 2 (correspondente a navegação nos seis sites analisados).

Verificando-se o processo de busca com o sistema (buscador Google) foi possível a identificação do caminho que o software de síntese de voz percorreu para o início da busca: sendo o direcionamento para a caixa de combinação editável ou para a barra de navegação de tarefa. A importância dessa verificação determina o que exatamente o usuário com cegueira pode compreender para a primeira etapa do processo de busca, que se trata do reconhecimento da página inicial do buscador. No tocante ao Nível 1, dois questionamentos foram levantados no campo da Percepção e Operacionalização, que se centraram na navegação pelos elementos da página dos resultados, que são: cabeçalho, submenu e a leitura de todos os elementos seguindo a ordem de tabulação.

Nesse contexto é possível identificar o detalhamento da navegação, ou seja, se o usuário com cegueira pode utilizar o comando de setas (acima e abaixo) para explorar todos os elementos da página, procurando explorar cabeçalhos, submenu e links com evidência nos resumos apresentados por cada resultado para ter noção das informações tratadas nos sites, ou se pode optar pela navegação com o uso da tecla $T A B$ em elementos focalizáveis. Como nesta pesquisa foram analisados dois termos compostos "Musicografia Braille" e "Eleições 2014", o buscador retornou uma média de 10 itens de resultados para primeira página. Desse modo, 
pode-se perceber até qual item o usuário pode estabelecer a sua busca, sabendo-se que pode realizar a análise do resumo da informação contida no site antes de adentrar.

Ainda nesse mesmo processo, verificou-se a possibilidade da leitura dos resultados da primeira página do buscador em conformidade com a horizontalidade e verticalidade. $\mathrm{Ou}$ seja, se o usuário pode estabelecer uma navegação por todos os resultados obtidos, ou se faz a leitura de cada resumo para então adentrar no site escolhido. É importante considerar que, para esta pesquisa, a análise foi realizada nos seis primeiros itens (sites) para cada busca realizada.

Referente ao Nível 2, que compreende a navegação nos sites, oito questões foram elaboradas segundo a simulação estabelecida nos campos da Percepção, Operacionalização e Compreensão. Nessa fase do processo de simulação, teve-se como base os princípios estabelecidos pela recomendação W3C, que se verificou com a possibilidade de identificação dos caracteres das páginas pelo software de síntese de voz, abrangendo a organização e estruturação dos sites. Nessa fase foi possível verificar a identificação do detalhamento da pesquisa que pode ser efetuada pelo usuário com cegueira em toda a página do site, ou se pode ocorrer o não estabelecimento da pesquisa por completo, seja pela falta de interesse nas informações apresentadas pelo site, ou por problemas de acessibilidade na navegação. Com isso, conseguiu-se analisar aspectos importantes como a leitura de conteúdos textuais e não textuais, assimilação da estrutura e dos conteúdos, possíveis dificuldades na utilização dos atalhos (em combinação com o teclado), tempo de leitura para o conteúdo da página, ambiguidade do conteúdo e sua legibilidade, e compreensão da operacionalização da página.

A partir do critério de Amplitude determinou-se a encontrabilidade da informação e possíveis desistências que possam ocorrer ao logo do processo de busca e navegação; nessa fase evidenciaram-se os campos da Percepção e Compreensão. Conforme os procedimentos estabelecidos nos Níveis 1 e 2, é possível entender se a recuperação da informação pode ser total ou se pode ocorrer desistência de busca em determinada página, que possa ocorrer devido à falta de interesse, ou pela falta de acesso aos conteúdos.

Com o critério de Tempo evidenciou-se o tempo do retorno do resultado do sistema de busca, o tempo possível que o usuário pode utilizar para o processo completo (busca, navegação e recuperação) e o tempo do retorno dos buscadores a partir do retorno sonoro com o software de síntese de voz. Sabe-se que o usuário com cegueira somente pode manipular o sistema computacional com a utilização de um sistema de síntese de voz que apreende informações acessíveis (textuais) a partir da interação com o sistema operacional, e as transformam em resposta sonora. Nesse sentido, o usuário sempre dependerá do sistema de síntese de voz para proceder aos comandos de utilização do sistema computacional.

A partir dessa observação, depreendeu-se em relação ao critério de tempo, que no processo de navegação web, para proceder aos comandos, o usuário estará dependente do carregamento do sistema de busca (buscador), e carregamento de páginas, para que o sistema de síntese de voz efetue o retorno sonoro desse carregamento. Nesse sentido, para a \begin{tabular}{l|l|l|l|l|l} 
(C) RDBCI: Rev. Digit. Bibliotecon. Cienc. Inf. & Campinas, SP & v.15 & n.1 & p. 211-233 & jan./abr. 2017 \\
\hline
\end{tabular} 
construção do checklist foi necessário considerar o tempo de carregamento do buscador e de cada página pesquisada (Tempo de Busca pelo Sistema - TBS), com o tempo de retorno sonoro do sistema de síntese de voz (Tempo de Retorno Sonoro - TRS).

De acordo com esta pesquisa, as estimativas de tempo identificadas, estabeleceram-se com os seguintes valores: aproximadamente $0,70 \mathrm{seg}$ para cada carregamento do buscador e páginas (TBS); e aproximadamente 2seg para o retorno sonoro do sistema de síntese de voz após cada carregamento (TRS). Com base nessas medições, em conjunto com o processo de busca efetuado por esta pesquisa, que considerou: busca, navegação e recuperação da informação desejada foi possível alcançar um tempo estimado para todo o tempo de pesquisa com o uso do sistema síntese de voz, considerando os termos utilizados: "Musicografia Braille" e "Eleições 2014"; e a acessibilidade encontrada em cada um dos seis sites (páginas) pesquisados. Desse modo, foi estimado o tempo de 50min para o tempo de pesquisa pelo usuário (TPU), considerado como aceitável para um procedimento de busca na primeira página de resultados, referente aos seis primeiros resultados recuperados, com boa acessibilidade ao acesso às informações. Desconsiderando a falta de acessibilidade encontrada ao longo do processo de busca.

Todos os questionamentos com relação ao critério de tempo correspondem ao campo da Operacionalização. É imprescindível compreender que o encaminhamento do processo de busca, navegação e recuperação da informação dependem do desempenho do software de síntese de voz, por isso a importância do entendimento do tempo que esse software atua em conjunto com o sistema de busca, e mesmo, na navegação nos sites, que determinarão a possibilidade de um processo de busca eficaz.

Com o critério de Satisfação constatou-se o possível sucesso que pode ser obtido com a efetivação da recuperação da informação desejada. Para esse critério contemplou-se o campo da Percepção. Considera-se que a utilização do software de síntese de voz é um dos elementos principais que evidenciará a satisfação da necessidade de informação de um usuário com cegueira. Os demais elementos referem-se à organização e estruturação em que se encontram os sistemas de busca, sites e páginas.

\section{CONSIDERAÇÕES}

As possibilidades existentes na atualidade, com a disponibilidade de diversas fontes de informação e o crescente e complexo fluxo informacional caracterizado pelo armazenamento e compartilhamento em rede, configuram-se como elementos essenciais de estudo para o entendimento do acesso à informação em ambiente web, onde se procura evidenciar e compreender as necessidades de informação existentes nos grupos de pessoas com cegueira, bem como entender se suas necessidades informacionais estão sendo supridas com eficácia. 
Os processos de busca, navegação e recuperação da informação, com base na utilização de dispositivos como softwares de síntese de voz, evidenciam uma realidade cuja proposta atual de acessibilidade ao acesso à informação, com as formas atuais de armazenamento e compartilhamento, compreende como uma das mais eficazes soluções para se obter a informação. Nesse sentido, procurar entender se esse processo é possível de ser desenvolvido de forma plena por todos os usuários com a mesma rapidez e eficácia foi exatamente a proposta desta pesquisa que buscou, no contexto das necessidades informacionais de pessoas com cegueira, compreender como se estabelece os processos de busca, navegação e recuperação da informação com base nas estratégias específicas utilizadas por essas pessoas em ambiente $w e b$, com ênfase na avaliação desse processo.

Sabe-se que as necessidades de informação de usuários com cegueira são inúmeras devido à falta de diversidade de suportes físico e digital, bem como de produção de materiais de informação em geral que possam vir a suprir as suas necessidades, considerando-se que o avanço das tecnologias assistivas centradas nos softwares de síntese de voz estão transformando o aspecto comportamental de buscar, navegar e recuperar a informação.

A proposta de construção de um checklist simples para avaliação de acessibilidade em procedimentos de busca, navegação e recuperação da informação possibilitou tanto apresentar algumas técnicas aplicadas no processo de navegação por uma pessoa com cegueira, como evidenciar as principais ocorrências de dificuldades nesse processo a partir da aplicação do sistema de síntese de voz, de maneira que se estruturou um instrumento básico que possa servir como modelo para checklists de acessibilidade gerais e específicos baseados na navegação web efetuada por usuários com cegueira.

Nesse contexto, os resultados alcançados com esta pesquisa permitiram estruturar um checklist com pontos básicos de verificação fundamentados no comportamento do sistema de síntese de voz, cuja aplicação oportunizou, tecnicamente, a observação de possíveis caminhos e dificuldades que precisam ser considerados na utilização desse sistema em pesquisas que tenham como propostas a construção de checklists de acessibilidade, gerais e específicos, com o apoio de usuários com cegueira; ou mesmo, observação, análise e avaliação de navegação web por esses usuários. Considerando-se que a eficácia desses tipos de checklists (gerais e específicos), na prática, somente será corporificada com base na participação e experiência do usuário com cegueira em seus processos de construção.

Dessa forma, o checklist elaborado por esta pesquisa, trata-se de um protótipo que visualiza a funcionalidade do software necessário a pessoas com cegueira, ou deficiência visual em geral, em processos de navegação web, que pode proporcionar a pesquisas futuras, a previsão do comportamento do sistema de síntese de voz, e mesmo, em determinados pontos de verificação, o comportamento do próprio usuário perante os caminhos a serem seguidos em processos de busca, navegação e recuperação da informação, subsidiando a observação e integração de novos elementos para a verificação de acessibilidade em ambiente web, de modo a se apresentar resultados e soluções que possam nortear futuros melhoramentos para o acesso à informação disponibilizada a todos.

\begin{tabular}{l|l|l|l|l|l}
\hline (C) RDBCI: Rev. Digit. Bibliotecon. Cienc. Inf. & Campinas, SP & v.15 & n.1 & p. 211-233 & jan./abr. 2017 \\
\hline
\end{tabular}




\section{LISTA DE VERIFICACIÓN DE ACCESIBILIDAD EN ENTORNOS WEB INFORMATIVO}

RESUMEN: Esta investigación se ocupa del proceso de búsqueda, navegación y recuperación de la información por la persona con ceguera en un entorno web, centrándose en el conocimiento de las áreas de recuperación y la arquitectura de la información, para comprender las estrategias utilizadas por estas personas para acceder a la información en la web. Su objetivo es proponer la construcción de una herramienta de accesibilidad, lista de control, comprobando que se utilizará para el análisis del comportamiento de la persona con ceguera en las acciones de búsqueda, sitios de navegación y recuperación y las páginas. Es un estudio exploratorio y descriptivo de la naturaleza investigación de carácter cualitativo, teniendo como metodología de investigación, estudio de casos, - la investigación para establecer un estudio específico de simulación de búsqueda, navegación y recuperación de información mediante el sistema de síntesis de voz, Acceso de escritorio no visual, en las ayudas técnicas de laboratorio, por razones de construcción de la lista de verificación para la comprobación de la accesibilidad. Se considera la fiabilidad de la investigación realizada y su importancia para la evaluación de la accesibilidad en el entorno web para mejorar el acceso a la información para las personas con la lectura limitada con el fin de ser utilizado en el análisis de comprobación de accesibilidad sitios y páginas web.

PALABRAS CLAVE: Recuperación de información. Accesibilidad a la información. Ceguera. Lista de verificación.

Submetido em: 29-06-2016

Aceito em: 10/11/2016

Publicado em: 21/11/2016 


\section{REFERÊNCIAS}

BARANAUSKAS, Maria Cecília Calani; SOUZA, Clarisse Sieckenius de. Desafio no 4 : acesso participativo e universal do cidadão brasileiro ao conhecimento. Computação Brasil, ano 8, n. 23, p. 7, 2006.

BERSCH, Rita. Introdução à tecnologia assistiva. Porto Alegre: CEDI, 2008.

BORGES, José Antonio dos Santos. Do braille ao DOSVOX: diferenças nas vidas dos cegos brasileiros. 2009. 343 f. Tese (Doutorado em Engenharia e Sistemas de Computação) Universidade Federal do Rio de Janeiro, Rio de Janeiro, 2009.

CHRISTENSEN, Lars Ballieu; STEVNS, Tanja. Biblus: a digital library to support integration of visually impaired in mainstream education. Springer, v. 7382, p. 36-42, 2012.

CRAVEN, Jenny; BROPHY, Peter. Non-Visual Access to the Digital Library (NoVA): the use of the digital library interfaces by blind and visually impaired people. Centre for Research in Library \& Information Management. The Manchester Metropolitan University. 2003.

DOWNEY, Laura; BANERJEE, Sumit. Building an information architecture checklist: encouraging and enabling IA from infrastructure to the user interface architecture. Journal of Information Architecture, v. 2, n. 2, p. 25-42, 2010.

DIAS, Claudia. Usabilidade na web: criando portais mais acessíveis. Rio de Janeiro: Alta Books, 2003.

GOLUB, Koraljka. Digital libraries and the blind and visually impaired. In: CARNET USERS CONFERENCE, 4., 2002, Zagreb. Anais... Zagreb, 2002.

HERNÁNDEZ SAMPIERI, Roberto; HERNÁNDEZ COLLADO, Carlos; BAPTISTA LUCIO, Pilar. Tipos de pesquisa. In: Metodologia da pesquisa. 3. ed. São Paulo:

McGraw-Hill, 2006. p. 96-115.

MELO, Amanda Meincke; BARANAUSKAS, Maria Cecília Calani. Design inclusivo de sistemas de informação na web. 2006. Disponível em:

<styx.nied.unicamp.br:8080/todosnos/.../texto_mini_curso_ihc2006.pdf>. Acesso em: 25 set. 2012.

MELO, Amanda Meincke. Design inclusivo de sistemas de informação na web. 2007. 349

f. Tese (Doutorado em Ciência da Computação) - Universidade Estadual de Campinas, Campinas, 2007.

MELO, Amanda Meincke; COSTA, Jean Braz da; SOARES, Sílvia Cristina de Matos. Tecnologias assistivas. In: PUPO, Deise Tallarico; MELO, Amanda Meincke; PÉREZ FERRÉS, Sofia. Acessibilidade: discurso e prática no cotidiano das bibliotecas. Campinas: UNICAMP, 2006. p. 62-70. 
PÉREZ FERRÉS, Sofia. Acessibilidade física. In: PUPO, Deise Tallarico; MELO, Amanda Meincke; PÉREZ FERRÉS, Sofia. Acessibilidade: discurso e prática no cotidiano das bibliotecas. Campinas: Unicamp/Biblioteca Central Cesar Lattes, 2008. p. 21-32.

MORVILLE, Peter; ROSENFELD, Louis. Information architecture for the world wide web. Sebastopol, CA: O' Reilly, 2006.

NASCIMENTO, Denise Morado. A abordagem sócio-cultural da informação. Inf. \& Soc.: Est., João Pessoa, v. 16, n. 2, p. 21-34, jul./dez. 2006.

NIELSEN, Jacob. iPad and Kindle Reading Speeds, 2010. Disponível em: <http://www.useit.com/alertbox/ipad-kindle-reading.html>. Acesso em: 07 mar. 2012.

RODRIGUES, Bruno Cesar; CRIPPA, Giulia. A recuperação da informação e o conceito de informação: o que é relevante em mediação cultural? Perspectivas em Ciência da Informação, v. 16, n. 1, p. 45-64, 2011.

ROGERS, Yvonne; SHARP, Helen; PREECE, Jennifer. Design de interação: além da interação homem-computador. Porto Alegre: Bookman, 2013.

SALES, Márcia Barros de. Desenvolvimento de um checklist para a avaliação de acessibilidade da web para usuários idosos. 2002. $121 \mathrm{f}$. Dissertação (Mestrado em Engenharia de Produção) - Universidade Federal de Santa Catarina, Florianópolis, 2002.

SANTOS, Lucy Souza; NASCIMENTO, Paulo Arthur Alves Santana do. EASY: tecnologia assistiva para mediar a educação a distância para os portadores de deficiência visual. 2006. 140 f. Monografia (Bacharelado em Informática) - Universidade Católica de Salvador, Salvador, 2006.

SARACEVIC, Tefko. Ciência da informação: origem, evolução e relações. Perspectivas em Ciência da Informação, Belo Horizonte, v.1, n.1, p. 41-62, jan./jun.1996.

ULIANA, Cleverson Casarin. NVDA: leitor de tela livre para windows. 2008. Disponível em: 〈http://acessibilidadelegal.com/33-nvda.php>. Acesso em: 27 set. 2014.

VENTURA, Magda Maria. O estudo de caso como modalidade de pesquisa. RevSOCERJ, v. 20, n. 5, p. 383-386, 2007.

WEI, Zhang; LIRONG, Song; CHUNMING, Li. An analysis of the development of China digital library for visual impairment website. The Electronic Library, v. 30, n. 6, p. 756763, 2012.

WORLD WIDE WEB CONSORTIUM. Web content accessibility guidelines 1.0. 1999. Disponível em: <http://www.w3.org/TR/WCAG10/>. Acesso em: 8 dez. 2014.

WORLD WIDE WEB CONSORTIUM. Recomendações de acessibilidade para conteúdo web 2.0. 2008. Disponível em: <http://www.ilearn.com.br/TR/WCAG20/>. Acesso em: 8 dez. 2014.

WURMAN, Richard Saul. Ansiedade de informação. São Paulo: Cultura, 1991.

\begin{tabular}{|c|c|c|c|c|c|}
\hline (C) RDBCI: Rev. Digit. Bibliotecon. Cienc. Inf. & Campinas, SP & v. 15 & n. & p. 211-233 & jan/abr. 2017 \\
\hline
\end{tabular}


WURMAN, Richard Saul. Information Architects. New York: Graphis, 1997.

YATACO MARÍN, Rosa María. Servicios bibliotecarios para personas condiscapacidad visual: el caso de la Sala para Invidentes "Delfina Otero Villarán” de laGran Biblioteca Pública de Lima. 2009. 94 f. Informe professional para optar el Título de Licenciado en Bibliotecología y Ciencias de laInformación - Universidad Nacional Mayor de San Marcos, Lima, 2009.

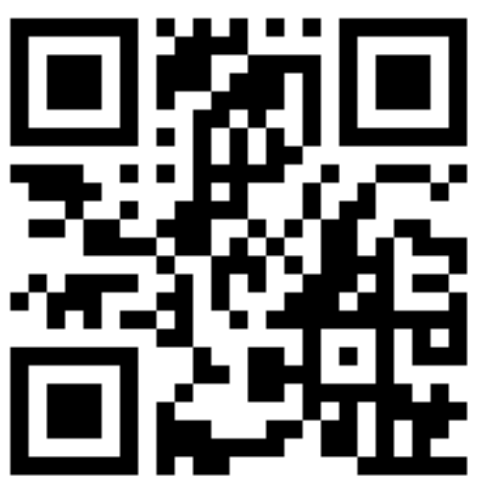

